\title{
Homocysteine reduces protein S-nitrosylation in endothelium
}

\author{
YULONG CHEN $^{1,2}$, SIHAI ZHAO ${ }^{1,2}$, YANLI WANG ${ }^{1,2}$, YAFENG LI $^{1,2}$, \\ LIANG BAI ${ }^{1,2}$, RUIHAN LIU ${ }^{1,2}$, JIANGLIN FAN ${ }^{3}$ and ENQI LIU ${ }^{1,2}$ \\ ${ }^{1}$ Research Institute of Atherosclerotic Disease, Xi'an Jiaotong University Cardiovascular Research Center; \\ ${ }^{2}$ Laboratory Animal Center, Xi'an Jiaotong University School of Medicine, Xi'an, Shaanxi 710061, P.R. China; \\ ${ }^{3}$ Department of Molecular Pathology, Interdisciplinary Graduate School of Medicine and Engineering, \\ University of Yamanashi, Yamanashi 409-3898, Japan
}

Received February 9, 2014; Accepted August 13, 2014

DOI: $10.3892 / \mathrm{ijmm} .2014 .1920$

\begin{abstract}
Hyperhomocysteinemia (HHcy) is a risk factor for cardiovascular disease. The S-nitrosylation of proteins is involved in the regulation of cardiovascular functions. However, whether homocysteine(Hcy) impairs vascularfunctions through the inhibition of protein S-nitrosylation in the endothelium remains to be determined. The experiments were performed in human umbilical vein endothelial cells (HUVECs). Male Sprague-Dawley rats, with or without administration of L-methionine, were used for the in vivo validation of findings. S-nitrosylation was analyzed using immunofluorescence for nitrosocysteine, and further confirmed by the biotin switch method. The levels of reactive oxygen species (ROS) were detected by 2',7'-dichlorofluorescein diacetate (DCFH-DA) staining. The levels of nitric oxide (NO) were determined by the nitrate reduction method. Protein expression was analysed by western blot analysis. The activity of nuclear factor $\kappa \mathrm{B}(\mathrm{NF}-\kappa \mathrm{B})$ was evaluated by an electrophoretic mobility shift assay (EMSA). The levels of plasma Hcy were measured by ELISA. The results showed that Hcy significantly reduced the levels of protein S-nitrosylation in HUVECs and endothelial S-nitrosylation of aorta. This reduction of protein S-nitrosylation was accompanied by increasing ROS, decreasing phosphorylation levels of Akt and endothelial nitric
\end{abstract}

Correspondence to: Professor Enqi Liu, Research Institute of Atherosclerotic Disease, Xi'an Jiaotong University Cardiovascular Research Center, Xi'an Jiaotong University School of Medicine, 76 Yanta West Road, Xi'an, Shaanxi 710061, P.R. China

E-mail: liuenqi@mail.xjtu.edu.cn

Abbreviations: DCF, 2',7'-dichlorofluorescein; EMSA, electrophoretic mobility shift assay; eNOS, endothelial nitric oxide synthase; Hcy, homocysteine; HUVECs, human umbilical vein endothelial cells; HHcy; hyperhomocysteinemia; NAC, N-acetylcysteine; $\mathrm{NO}$, nitric oxide; NF- $\kappa \mathrm{B}$, nuclear factor $\kappa \mathrm{B}$; ROS, reactive oxygen species; GSNO, S-nitrosoglutathione

Key words: homocysteine, endothelial cells, nitric oxide, S-nitrosylation oxide synthase (eNOS), and reduced levels of nitric oxide in HUVECs. In addition, it was found that Hcy increased the protein expression of vascular cell adhesion molecule-1 by attenuating the cytoplasm S-nitrosylation of NF- $\mathrm{kB}$ (p65). These data suggested that Hcy impairs endothelial functions by inhibiting endothelial protein S-nitrosylation.

\section{Introduction}

S-nitrosylation is a protein modification in which a nitrosyl group covalently attaches to the thiol group on protein cysteine residues, forming an S-nitrosothiol. S-nitrosylation is a redox mechanism for the dynamic and post-translational regulation of most or all of the major classes of proteins and is independent of enzyme catalysis, labile modification, and on/off switch-like photophosphorylation, involved in cellular signal transduction (1-3). The S-nitrosylation of proteins is involved in the regulation of cardiovascular functions (2). Classically, nitric oxide (NO) is generated through the activation of endothelial NO synthase (eNOS), and NO diffuses from the endothelial cells to the vascular smooth muscle cells, where it binds to its intracellular receptor, soluble guanylyl cyclase, and activates protein kinase $\mathrm{G}$ to mediate vasorelaxation (1-3). However, NO also regulates widespread and diverse biologic functions in the endothelium, such as ion channel activity (4), antioxidative stress (5), anti-apoptotic mechanisms (6); and anti-inflammatory responses $(3,7)$. These functions have been attributed to the protein S-nitrosylation (3-7). It has been shown that many factors are involved in the regulation of protein S-nitrosylation in endothelial cells. Tumor necrosis factor- $\alpha$ (8), oxidized low-density lipoprotein (8); and high glucose (9) decrease protein S-nitrosylation, whereas shear stress (10), 17b-estradiol (3); and acute hypoxia (11) upregulate protein S-nitrosylation in endothelial cells.

Homocysteine (Hcy) is a sulfur-containing amino acid formed during the transmethylation of methionine. High plasma levels of Hcy, termed hyperhomocysteinemia (HHcy), is an independent risk factor for cardiovascular disease. Elevated plasma levels of Hcy are found in 5-10\% of the general population and in up to $40 \%$ of patients with vascular disease (12). It has been reported that Hcy reduced the levels of NO (13). However, whether Hcy also impairs protein 
S-nitrosylation in endothelial cells by mediating the levels of NO remains to be determined. Thus, we hypothesized that Hcy may decrease the levels of protein S-nitrosylation in endothelial cells. In the present study, we examined the effects of Hcy on human umbilical vein endothelial cells (HUVECs) and rat aortas. The results showed that Hcy significantly reduced the cellular protein S-nitrosylation. Molecular mechanisms that are involved in the process of S-nitrosylation were also examined. These findings provide new insights into understanding of the pathway for Hcy-mediated vascular damage.

\section{Materials and methods}

Cell culture. HUVECs were isolated from human umbilical cords. The investigation conformed to the principles outlined in the Declaration of Helsinki for the use of human tissue or subjects. The HUVECs were isolated using $0.1 \%$ (w/v) collagenase (Gibco/Invitrogen, Carlsbad, CA, USA). The cells were collected and cultured in M199 medium supplemented with $20 \%$ fetal bovine serum (FBS) (both from Gibco/Invitrogen), and $1 \%(\mathrm{v} / \mathrm{v})$ endothelial cell growth supplement (ScienCell Research Laboratories, Carlsbad, CA, USA) at $37^{\circ} \mathrm{C}$ in a humidified atmosphere of $5 \% \mathrm{CO}_{2}$ and $95 \%$ air. The growth medium was changed every three days until the cells reached confluence. The identity of the HUVECs was performed by positive staining with an anti-von Willebrand factor (vWF) antibody (Thermo Fisher Scientific/Pierce, Rockford, IL, USA) (3). After the HUVECs became confluent, they were incubated in M199 medium containing 2\% FBS for $16 \mathrm{~h}$ prior to different treatments.

Animal model. Moderate HHcy was induced in male Sprague-Dawley rats (180-200 g; supplied by the Laboratory Animal Center of Xi'an Jiaotong University, Xi'an, China) $(\mathrm{n}=12)$ by oral administration of L-methionine $(1 \mathrm{~g} / \mathrm{kg}$ body weight per day) (Fluka/Sigma-Aldrich, St. Louis, MO, USA) and succinylsulfathiazole $(0.5 \mathrm{~g} / \mathrm{kg}$ body weight per day $)$ (Fluka/Sigma-Aldrich) for 4 weeks as previously described (14). The animal experiments in this study were approved by the Laboratory Animal Administration Committee of Xi'an Jiaotong University and performed according to the Guidelines for Animal Experimentation of Xi'an Jiaotong University, the Guidelines on the Care and Use of Laboratory Animals issued by the Chinese Council on Animal Research, and the Guide for the Care and Use of Laboratory Animals published by the US National Institutes of Health (NIH publication no. 85-23, revised 1996).

Reactive oxygen species (ROS) assay. Intracellular ROS generation was assessed using 2',7'-dichlorofluorescein diacetate (DCFH-DA). ROS in the cells oxidize DCFH-DA, yielding the fluorescent compound 2',7'-dichlorofluorescein (DCF). The levels of ROS were detected using the ROS assay kit (Beyotime Institute of Biotechnology, Nantong, China).

Nitrate/nitrite assay. The levels of NO of the conditioned medium were determined by estimating the total concentration of nitrate/nitrite using a nitrate/nitrite colorimetric assay kit (Jiancheng Technology Co., Ltd., Nanjing, China).
Plasma Hcy assay. The rats were anesthetized with an intraperitoneal injection of sodium pentobarbital $(50 \mathrm{mg} / \mathrm{kg})(14)$. The blood was collected in centrifuge tubes (containing EDTA) from the aortas of fasted rats. The blood was immediately cooled on ice and centrifuged at 3,000 x g for $20 \mathrm{~min}$ at $4^{\circ} \mathrm{C}$. Plasma was then stored at $-20^{\circ} \mathrm{C}$ until assayed. Total plasma Hcy concentrations were measured using an ELISA kit (R\&D Systems, Inc., Minneapolis, MN, USA).

Immunofluorescence. The HUVECs were fixed with $4 \%$ paraformaldehyde for $15 \mathrm{~min}$ at room temperature. The cells were permeabilized with $0.1 \%$ (v/v) Triton X-100 for $15 \mathrm{~min}$, and blocked with $5 \%$ horse serum for $30 \mathrm{~min}$, and incubated with anti-nitrosocysteine rabbit polyclonal antibody (Abcam, Cambridge, MA, USA) or anti-p65 antibody (Santa Cruz Biotechnology, Inc., Santa Cruz, CA, USA) at $4^{\circ} \mathrm{C}$. After overnight incubation the cells were treated with a FITC-conjugated goat anti-rabbit IgG (Santa Cruz Biotechnology, Inc.) for $30 \mathrm{~min}$ at room temperature. Nuclei were stained with 4,6-diamidino-phenylindole (DAPI; $0.2 \mathrm{mg} / \mathrm{ml}$ in $10 \mathrm{mM}$ Tris-HCl, pH 7.0, $10 \mathrm{mM}$ EDTA, $100 \mathrm{mM} \mathrm{NaCl}$ ). To validate the anti-S-nitrosocysteine antibody, we treated HUVECs for 10 min with $1 \mathrm{mM}$ DL-dithiothreitol (DTT), which caused severe loss of S-nitrosocysteine staining. For the negative control, fixed and permeabilized cells were preincubated with $0.8 \% \mathrm{HgCl}_{2}$ for $1 \mathrm{~h}$ at $37^{\circ} \mathrm{C}(8)$.

To stain the aorta, $10-\mu \mathrm{m}$ sections were placed on glass slides and fixed for $10 \mathrm{~min}$ in acetone at room temperature. After permeabilization, the sections were incubated with rabbit polyclonal anti-nitrosocysteine antibody and a mouse monoclonal anti-vWF antibody overnight at $4^{\circ} \mathrm{C}$. The incubation with a TRITC-conjugated goat anti-rabbit IgG $(\mathrm{H}+\mathrm{L})$ (Santa Cruz Biotechnology) and a FITC-conjugated goat anti-mouse $\operatorname{IgG}(\mathrm{H}+\mathrm{L})$ was performed. Nuclei were stained with DAPI. The results were visualized using fluorescence microscopy (Olympus, Tokyo, Japan). The mean fluorescence intensity was analyzed by Image-Pro Plus 6.0 software (Media Cybernetics Inc., Bethesda, MD, USA) (3).

Detection of S-nitrosylated proteins by the biotin switch assay. The HUVECs were lysed in HEN buffer (100 mM Hepes, $1 \mathrm{mM}$ EDTA, and $0.1 \mathrm{mM}$ neocuproine, $\mathrm{pH}$ 8.0) containing $1 \%(\mathrm{w} / \mathrm{v})$ SDS and $1 \mathrm{mM}$ PMSF plus protease inhibitors (Hoffmann-La Roche, Basel, Switzerland). The proteins extracted from the HUVECs were quantified using a BCA assay kit (Thermo Fisher Scientific/Pierce). Equal amounts of protein were incubated with HEN buffer containing $2.5 \%(\mathrm{w} / \mathrm{v}) \mathrm{SDS}$ and $0.1 \%(\mathrm{v} / \mathrm{v}) \mathrm{S}$-methyl-methanethiosulfonate (Fluka/Sigma-Aldrich) at $50^{\circ} \mathrm{C}$ in the dark for $20 \mathrm{~min}$. The extracts were precipitated with cold acetone. The proteins were re-suspended in HEN buffer plus 1\% SDS. S-nitrosothiols were decomposed by adding ascorbate followed by incubation with $2.5 \mathrm{mg} / \mathrm{ml}$ biotin-HPDP (Thermo Fisher Scientific/Pierce) for $1 \mathrm{~h}$ at room temperature. The proteins were subsequently precipitated again using acetone and resuspended in non-reducing Laemmli loading buffer. For purification of the biotinylated proteins, the proteins precipitated by acetone were diluted with neutralization buffer [20 mM HEPES (pH 7.7, $100 \mathrm{mM} \mathrm{NaCl}, 1 \mathrm{mM}$ EDTA, and $0.5 \%$ (v/v) Triton X-100] and $50 \%$ streptavidin agarose suspension (Fluka/Sigma-Aldrich) 
and incubated for $1 \mathrm{~h}$ at room temperature. The proteins were eluted with elution buffer.

Samples from the biotin switch assay were separated on $12 \%$ SDS polyacrylamide gels and transferred to PVDF membranes (EMD Millipore, Billerica, MA, USA). PVDF membranes were blocked with $5 \%$ non-fat dried milk for $1 \mathrm{~h}$ at $37^{\circ} \mathrm{C}$ and incubated with specific antibodies, including horseradish peroxidase-conjugated anti-biotin (Cell Signaling Technology, Danvers, MA, USA), anti- $\beta$-actin antibody (Abcam), and anti-p65 antibody, overnight at $4^{\circ} \mathrm{C}$. Membranes were then incubated with a horseradish peroxidase-conjugated goat against mouse IgG $(\mathrm{H}+\mathrm{L})$, followed by enhanced chemiluminescence using the SuperSignal West Pico Substrate kit (both from Thermo Fisher Scientific/Pierce). The protein bands were detected and analyzed using the Chemi-Doc-it HR 410 imaging system (Ultra-Violet Products, Ltd., Upland, CA, USA) (15).

Western blot analysis. The cells were lysed on ice for $1 \mathrm{~h}$ in RIPA buffer [50 mM Tris- $\mathrm{HCl}$ (pH 8.0), $150 \mathrm{mM} \mathrm{NaCl}$, $1 \%$ Triton $\mathrm{X}-100(\mathrm{v} / \mathrm{v}), 1 \%$ deoxycholic acid (w/v), and $0.1 \%$ sodium dodecyl sulfate)] containing $0.5 \mathrm{mM}$ PMSF and protease inhibitors (Hoffmann-La Roche). The protein concentration was measured.Each sample was subsequently denatured by boiling for $10 \mathrm{~min}$ in Laemmli loading buffer. Equal amounts of protein were separated by $12 \%$ SDS-polyacrylamide gel electrophoresis and transferred to PVDF membranes. After blocking with $5 \%$ non-fat dried milk for $1 \mathrm{~h}$ at $37^{\circ} \mathrm{C}$, the membranes were incubated overnight at $4^{\circ} \mathrm{C}$ with the following antibodies: anti-phospho-Akt (Ser-473) antibody, anti-phospho-eNOS (Ser-1177) antibody (Cell Signaling Technology), anti-Akt antibody, anti-eNOS antibody (Abcam), anti-vascular cell adhesion molecule-1 (VCAM-1) antibody (Santa Cruz Biotechnology, Inc.), and anti- $\beta$-actin antibody. After washing, the membranes were incubated using horseradish peroxidase-conjugated goat anti-mouse or anti-rabbit $\operatorname{IgG}$ (Thermo Fisher Scientific/Pierce) for $1 \mathrm{~h}$ at $37^{\circ} \mathrm{C}$, followed by enhanced chemiluminescence using the SuperSignal West Pico Substrate kit. The protein bands were analyzed using the Chemi Doc-it HR 410 imaging system (16).

Electrophoretic mobility shift assay (EMSA). Nuclear and cytoplasmic protein extraction were investigated. NE-PER Nuclear and Cytoplasmic Extraction Reagents (Thermo Fisher Scientific/Pierce) were used to extract the cell proteins according to the manufacturer's instructions. The nuclear proteins were quantified and stored at $-80^{\circ} \mathrm{C}$ until they were used for the EMSA.

Nuclear factor $\kappa B(N F-\kappa B)$ DNA-binding activity. The EMSA was performed with IRDye 700 infrared dye-labeled double-stranded oligonucleotides containing an $\mathrm{NF}-\kappa \mathrm{B}$ consensus site (5'-AGT TGA GGG GAC TTT CCC AGG C-3',

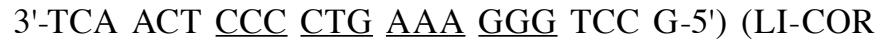
Biosciences, Lincoln, NE, USA). The nuclear extracts $(8 \mu \mathrm{g}$ per reaction) and $50 \mathrm{fmol}$ of the oligonucleotides were preincubated in $20 \mu \mathrm{l}$ binding buffer $[10 \mathrm{mM}$ Tris- $\mathrm{HCl}(\mathrm{pH} 7.5)$, $50 \mathrm{mM} \mathrm{NaCl}, 3 \mathrm{mM}$ DTT, $0.5 \mu \mathrm{g}$ poly dI-dC, $5 \mathrm{mM} \mathrm{MgCl}_{2}$, and $0.1 \%$ Tween-20)] for $30 \mathrm{~min}$ at room temperature. The specificity of the bands was confirmed by supershifting with specific antibodies of p65 and p50 (Santa Cruz Biotechnology, Inc.) and by competition with $100 \mathrm{x}$ specific cold competitor (100 x SPC) and $100 \times$ non-specific cold competitor (100 x NSC) (LI-COR Biosciences). The DNA protein complexes were separated on $5 \%$ native polyacrylamide gels for $60 \mathrm{~min}$ at $100 \mathrm{~V}$ in $0.5 \mathrm{X}$ TBE buffer. The signals were scanned and quantified using the Odyssey infrared imaging system (LI-COR Biosciences) (17).

Statistical analysis. Data are presented as the means \pm SEM. One-way ANOVA followed by an LSD test and Student's t-tests were used for the comparison between the groups. $\mathrm{P}<0.05$ was considered to be statistically significant. The analysis was performed using the SPSS 13.0 software (SPSS Inc., Chicago, IL, USA).

\section{Results}

Hcy reduces protein S-nitrosylation in HUVECs. HUVECs were treated with different doses of Hcy $(0.125,0.5$ and $2 \mathrm{mM})$ for $24 \mathrm{~h}$. Cell morphology was normal following treatment with Hcy (data not shown). The protein S-nitrosylation was detected for S-nitrosocysteine using immunofluorescence and was further confirmed by the biotin switch method. We found that treatment with different doses of Hcy $(2,0.5$ and $0.125 \mathrm{mM})$ resulted in the reductions $(44>34>8 \%$, respectively) in the protein $\mathrm{S}$-nitrosylation compared with the control group (Fig. 1A and B). However, there were no significant differences between $0.125 \mathrm{mM}$ and the control. Protein S-nitrosylation was significantly decreased in HUVECs treated with Hcy $(0.5$ or $2 \mathrm{mM})$. In addition, we found that S-nitrosocysteine was present in the nuclei and cytosol of the HUVECs (Fig. 1A). The biotin switch method on HUVECs further confirmed our finding (Fig. 1C).

Hyperhomocysteinemia attenuates endothelial S-nitrosylation of aortas in the rat. We assessed whether HHcy promoted a decrease in S-nitrosylation levels in the endothelium. HHcy was induced in rats by feeding a high-methionine diet to male Sprague-Dawley rats. As a result, the high-methionine diet resulted in a significant 3.6-fold increase in the plasma Hcy levels (17.2 vs. $4.8 \mathrm{mM}$ in control rats) (Fig. 2C). Furthermore, we detected a significant decrease in nitrosocysteine staining in the endothelium of thoracic aorta sections of L-methionine-fed rats compared with the controls (Fig. 2A and B).

ROS scavengers reverse the effect of Hcy on protein S-nitrosylation. ROS are involved in the deleterious effects of Hcy on endothelial functions (13). It is unclear whether Hcy reduces protein S-nitrosylation by ROS. The results showed that Hcy significantly increased ROS levels. This effect was blocked by vitamin $\mathrm{C}$ (VitC) and $\mathrm{N}$-acetylcysteine (NAC), which are both ROS scavengers (18) (Fig. 3A and B). Furthermore, VitC and NAC significantly increased the decreased levels of protein S-nitrosylation reduction by Hcy (Fig. 3C and D), suggesting that ROS play a crucial role in the Hcy-induced reduction of protein S-nitrosylation in endothelial cells.

Furthermore, the results showed that protein S-nitrosylation levels were partially restored in the Hcy-treated endothelial cells in the presence of the NO donor S-nitrosoglutathione (GSNO). 

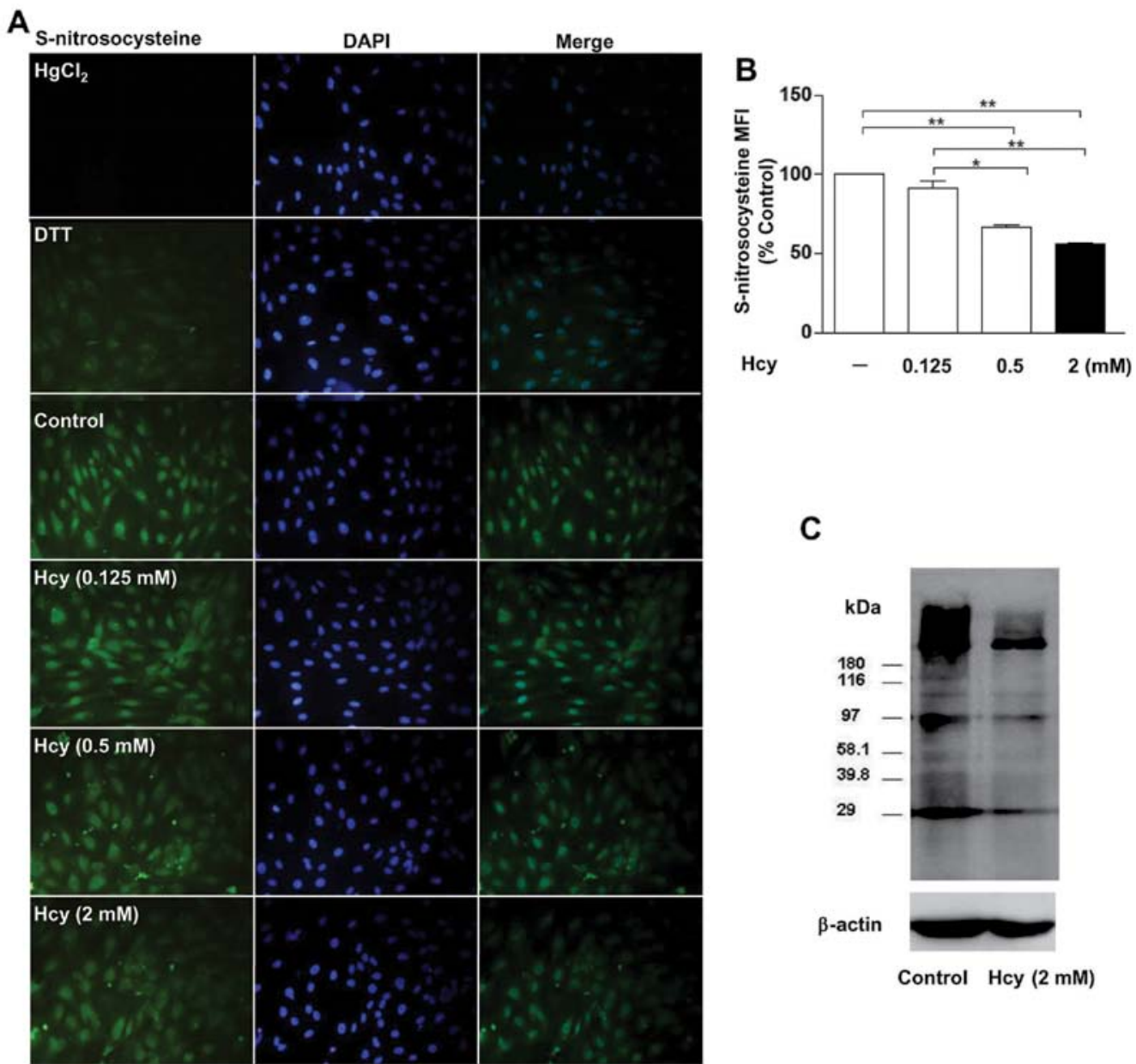

Figure 1. Homocysteine (Hcy) attenuated protein S-nitrosylation in human umbilical vein endothelial cells (HUVECs). HUVECs were exposed to different doses of Hcy $(0.125,0.5$ and $2.0 \mathrm{mM})$ for $24 \mathrm{~h}$. (A and B) The S-nitrosylated proteins in intact cells were detected by immunofluorescence for nitrosocysteine. (C) Representative western blot analysis showing biotinylated bands in control and Hcy-treated ( $2 \mathrm{mM}, 24 \mathrm{~h}$ ) HUVEC lysates that underwent the biotin switch assay. The means \pm SEM of three independent experiments are presented. ${ }^{*} \mathrm{P}<0.05$ and ${ }^{* *} \mathrm{P}<0.01$, respectively. MFI, mean fluorescence intensity.
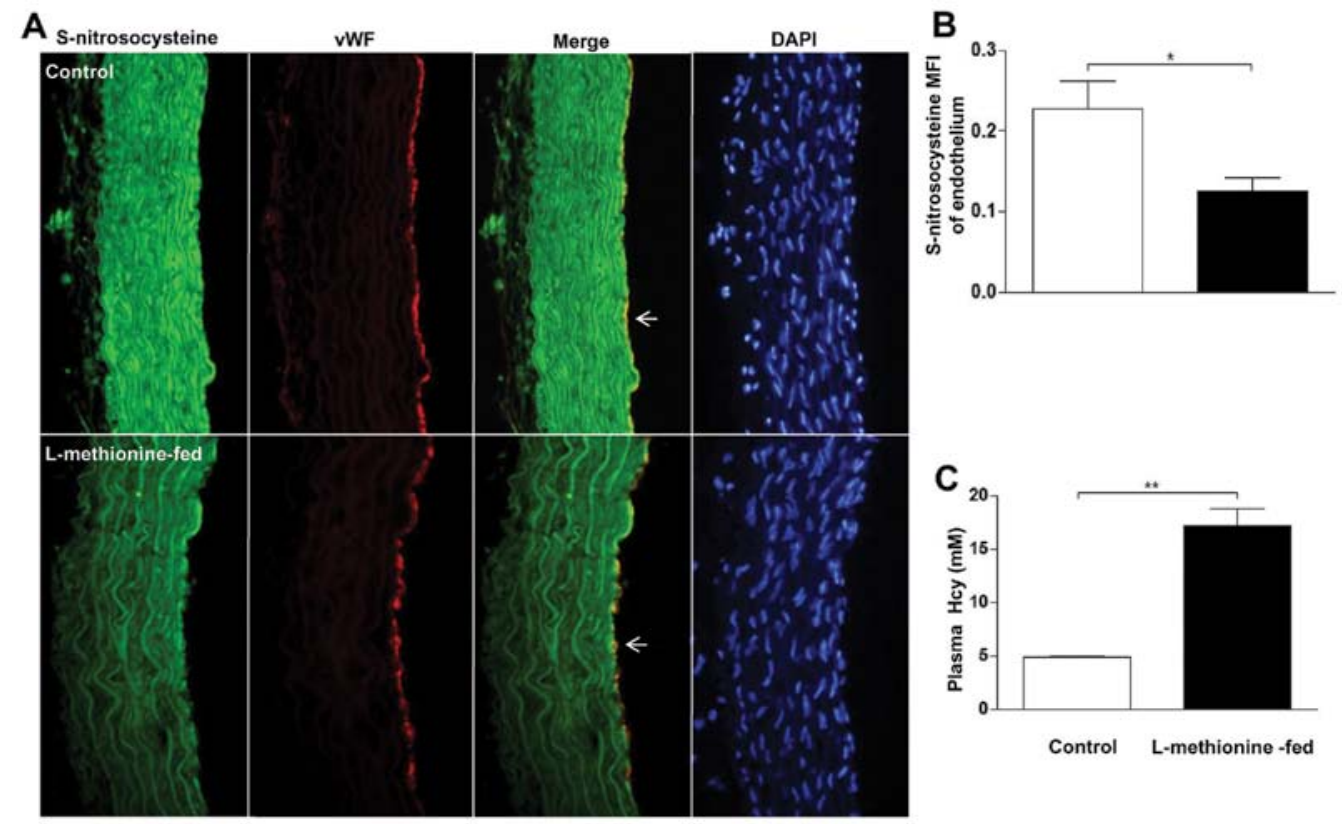

Figure 2. Hyperhomocysteinemia (HHcy) attenuated endothelial S-nitrosylation of the aorta in the rat model. Endothelial protein S-nitrosylation was observed by double immunofluorescence labeling for nitrosocysteine (green) and von Willebrand factor (vWF) (red, endothelium). (A and B) The nuclei were stained with 4,6-diamidino-phenylidole (DAPI) in rat aorta. (C) The plasma Hcy levels in rats were measured as shown in. Data from six rats in each group are presented as the means \pm SEM. MFI, mean fluorescence intensity. Arrow shows endothelial S-nitrosylation of the aorta. 

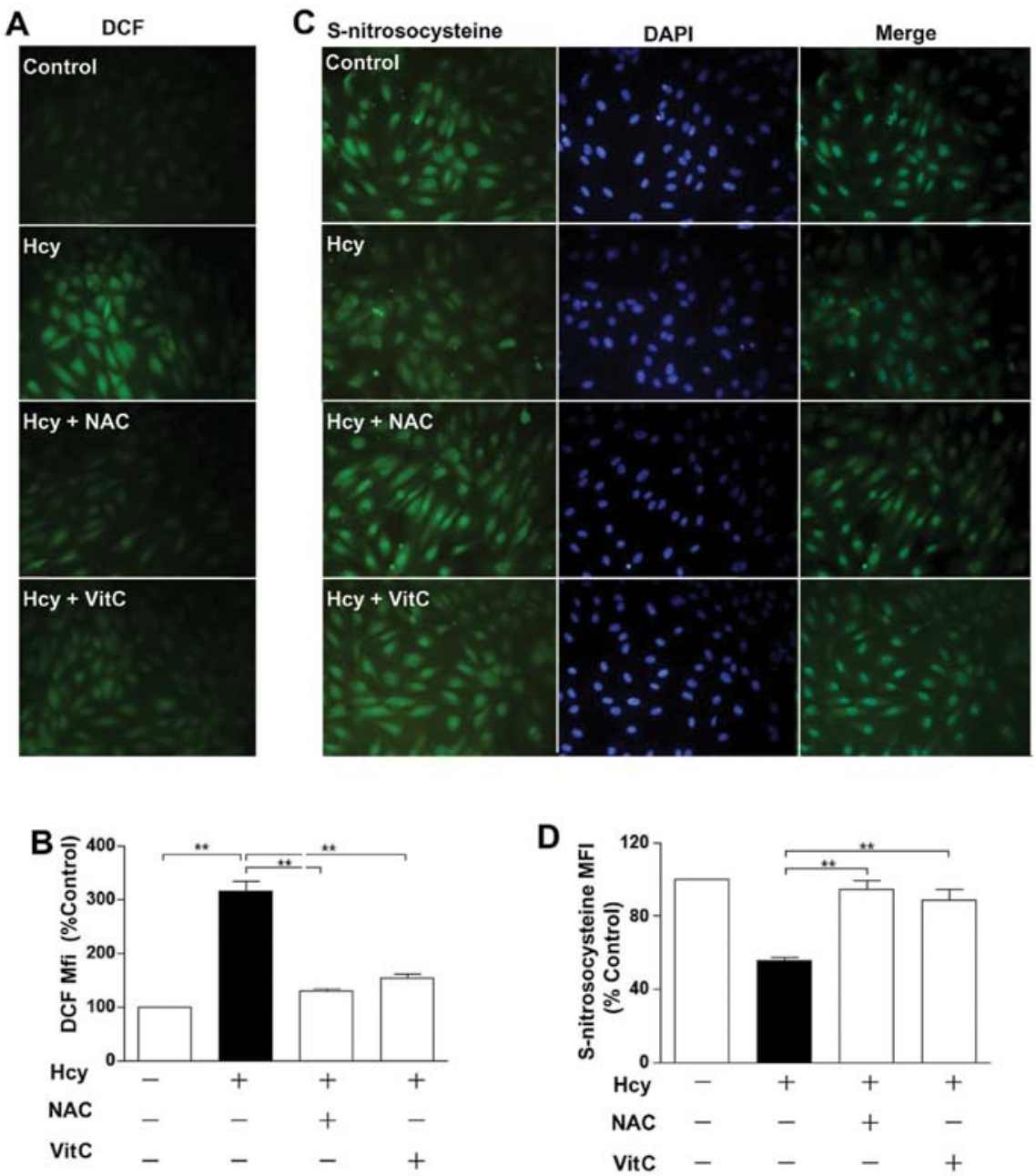

Figure 3. Reactive oxygen species (ROS) scavengers reversed the effect of homocysteine (Hcy) on protein S-nitrosylation in human umbilical vein endothelial cells (HUVECs). HUVECs were treated with $2 \mathrm{mM} \mathrm{Hcy}$ with or without vitamin C (VitC) (100 $\mu \mathrm{M})$ or N-acetylcysteine (NAC) (1 mM) for 24 h. (A and B) ROS were determined using DCF staining. (C and D) The S-nitrosylated proteins in intact cells were detected by immunofluorescence for nitrosocysteine. The means \pm SEM of three independent experiments are presented. ${ }^{* *} \mathrm{P}<0.01$. MFI, mean fluorescence intensity.

However, these levels of S-nitrosylation were abrogated by DTT (Fig. 4A and B). These data suggested that Hcy reduces protein S-nitrosylation by decreasing the levels of NO.

Involvement of oxidative stress and the Akt/eNOS/NO signaling pathway. We investigated whether the Akt/eNOS/NO pathway is involved in Hcy-induced protein S-nitrosylation in endothelial cells. The protein levels of Akt and eNOS were not affected by Hcy. (19). Thus, after treatment with Hcy, we determined the phosphorylation of Akt at Ser-473 and eNOS at Ser-1177 and the NO levels of cell-free supernatants in HUVECs. The results showed that Hcy downregulated the phosphorylation of Akt at Ser-473 and eNOS at Ser-1177 and decreased the levels of NO, while the effects were partially reversed by the addition of VitC (Fig. 5A-C). Furthermore, the restoration of protein S-nitrosylation by VitC was abrogated by the LY294002 (PI3K/Akt inhibitor) and L-NAME (NOS inhibitor; it reversibly inhibits eNOS activity) (Fig. 6A-C). These results suggested that Hcy increases the levels of intracellular ROS and subsequently decreases NO production by suppressing the Akt/eNOS/NO signaling pathway, which inhibits protein S-nitrosylation in HUVECs.
Hcy upregulates $N F-\kappa B$ activity. To investigate whether Hcy-induced protein S-nitrosylation affects NF- $\kappa$ B activity, we detected the levels of S-nitrosylation and the activity of $N F-\kappa B$. The results showed that Hcy abrogated NF- $\kappa$ B S-nitrosylation, which was accompanied by an increase in the activity of NF- $\kappa \mathrm{B}$ (Fig. 7A-D).

Hcy effect on levels of S-nitrosylation of p65 and VCAM-1. To determine the relationship between $\mathrm{S}$-nitrosylated NF- $\kappa \mathrm{B}(\mathrm{p} 65)$ and VCAM-1, our data showed that Hcy increased the protein expression of VCAM-1, whereas GSNO blocked the increase of VCAM-1 protein levels induced by Hcy, and DTT restored the expression of VCAM-1 (Fig. 8). However, the expression of VCAM-1 is upregulated along with the levels of cytoplasm S-nitrosylated NF- $\mathrm{B}$ (p65) decreasing (Fig. 4A and B).

\section{Discussion}

To the best of our knowledge, the present study has demonstrated for the first time that, Hcy attenuated the protein S-nitrosylation levels in HUVECs and an in vivo model. Attenuation of the S-nitrosylation induced by Hcy was mediated by the ROS/Akt/ 
A

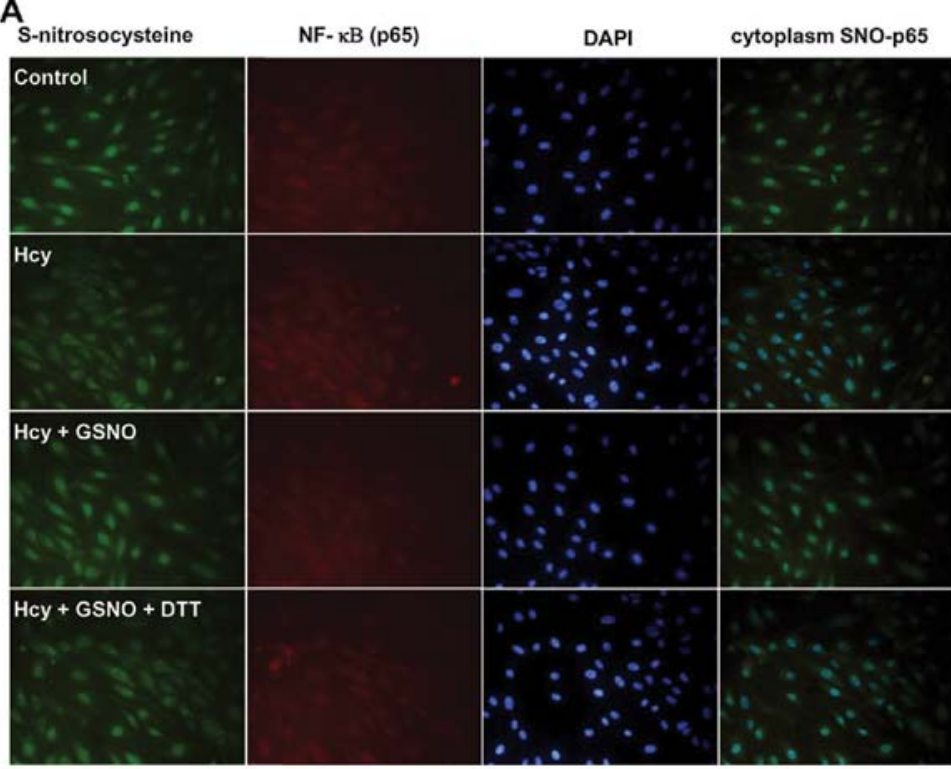

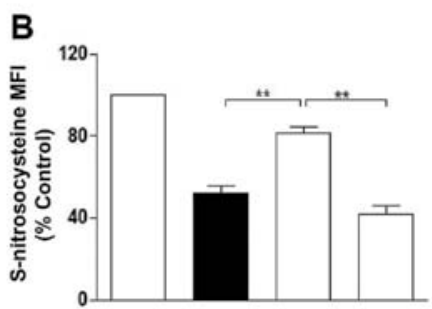

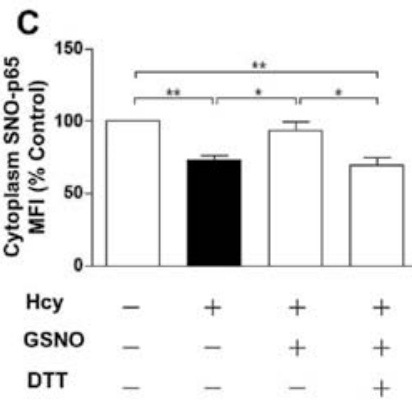

Figure 4. Effect of homocysteine (Hcy) on protein S-nitrosylation and nuclear factor $\kappa \mathrm{B}$ (NF- $\kappa \mathrm{B})$ S-nitrosylated in human umbilical vein endothelial cells (HUVECs). HUVECs were treated with $2 \mathrm{mM}$ Hcy and treated or not treated with nitric oxide (NO) donor S-nitrosoglutathione (100 $\mu \mathrm{M})$ for $24 \mathrm{~h}$. A portion of the cells was incubated with DL-dithiothreitol (DTT) $(1 \mathrm{mM})$ for $10 \mathrm{~min}$ and washed twice. (A and B) The S-nitrosylated proteins in the intact cells were detected by immunofluorescence for nitrosocysteine. (A and C) The S-nitrosylated NF- $\mathrm{kB}$ ( $\mathrm{p} 65$ ) in the intact cells was detected by double immunofluorescence labeling for nitrosocysteine and NF- $\kappa B$ (p65). The means \pm SEM of three independent experiments are presented. ${ }^{*} \mathrm{P}<0.05$ and ${ }^{* *} \mathrm{P}<0.01$, respectively. MFI, mean fluorescence intensity.

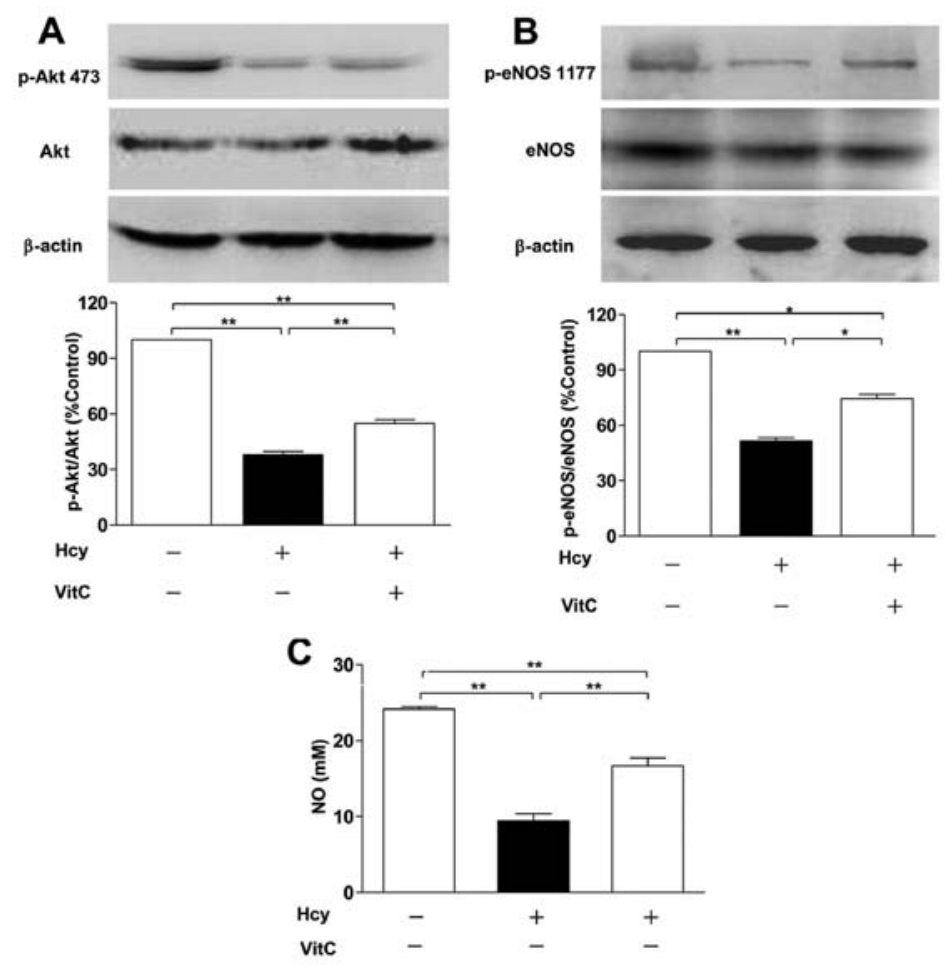

Figure 5. Effect of homocysteine (Hcy) on the reactive oxygen species (ROS)/Akt/endothelial nitric oxide (NO) synthase (eNOS)/NO signaling pathway in human umbilical vein endothelial cells (HUVECs). HUVECs were treated with $2 \mathrm{mM}$ Hcy and treated or not treated with vitamin C (VitC) (100 $\mu \mathrm{M})$ for $24 \mathrm{~h}$. (A) The levels of phosphorylation of Akt at Ser-473 in the cell lysates were detected using western blot analysis. (B) The levels of phosphorylation of eNOS at Ser-1177 in the cell lysates were detected using western blot analysis. (C) The levels of nitrate/nitrite in the cell supernatants were measured. The means \pm SEM of three independent experiments are presented. ${ }^{*} \mathrm{P}<0.05$ and ${ }^{* *} \mathrm{P}<0.01$, respectively.

eNOS/NO signaling pathway. Hcy induces ROS production, followed by the inhibition of Akt and eNOS phosphorylation. As a result, the reduced intracellular NO leads to the inhibition of protein S-nitrosylation. Moreover, we found that Hcy may upregulate the expression of VCAM-1 via the reduction of cytoplasm S-nitrosylation of NF- $\mathrm{kB}$ in endothelial cells. 
$\mathbf{A}_{\mathrm{s}+\mathrm{n}}$

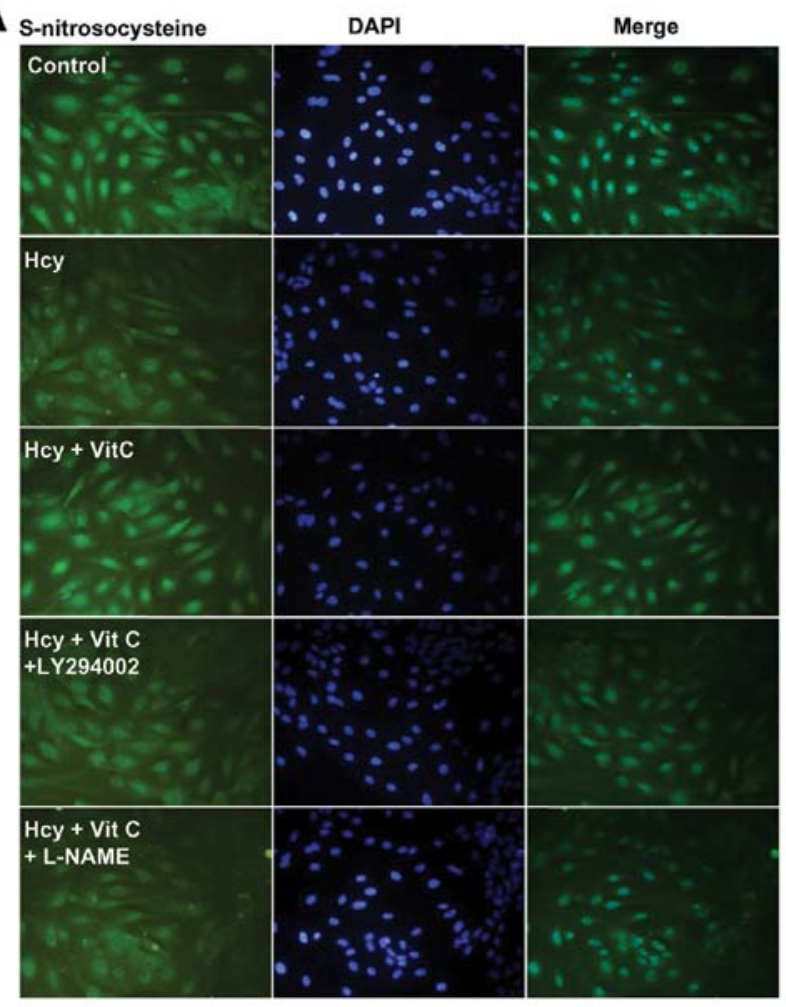

B

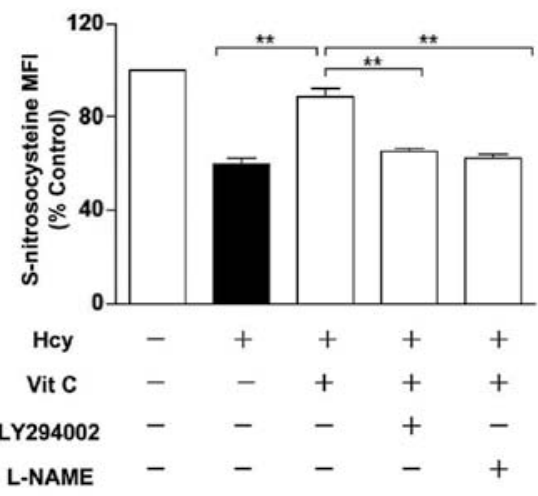

Figure 6. Homocysteine (Hcy) attenuated protein S-nitrosylation via the reactive oxygen species (ROS)/Akt/endothelial nitric oxide (NO) synthase (eNOS) signaling pathway in human umbilical vein endothelial cells (HUVECs). HUVECs were treated with LY294002 (10 $\mu \mathrm{M})$ or L-NAME (100 $\mu \mathrm{M}$ ) for 30 min prior to incubation with Hcy $(2 \mathrm{mM})$ and vitamin $\mathrm{C}$ (VitC) $(100 \mu \mathrm{M})$ for $24 \mathrm{~h}$. The S-nitrosylated proteins in intact cells were detected by immunofluorescence for nitrosocysteine $(\mathrm{A}$ and $\mathrm{B})$. The means $\pm \mathrm{SEM}$ of three independent experiments are presented. ${ }^{* *} \mathrm{P}<0.01$. MFI, mean fluorescence intensity.

A

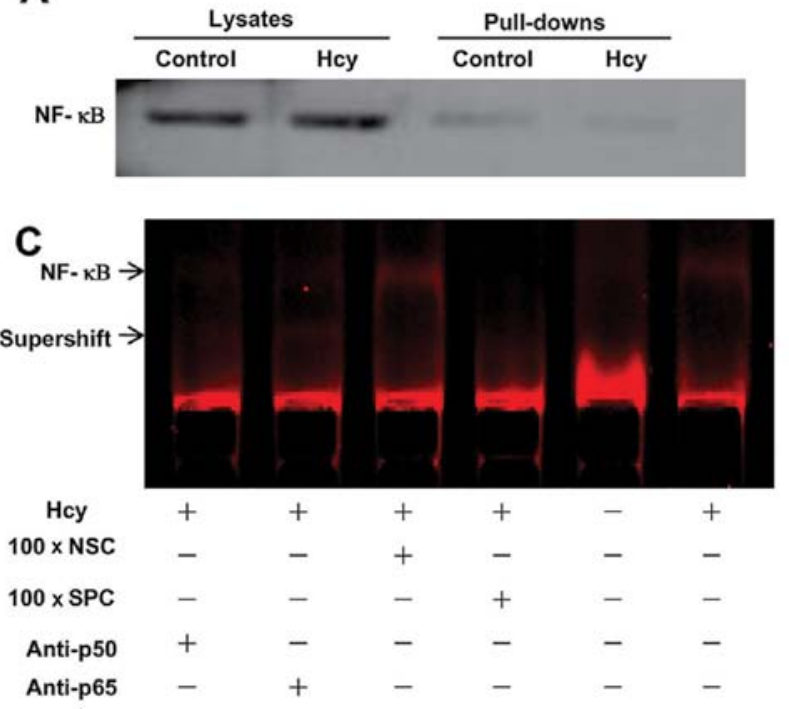

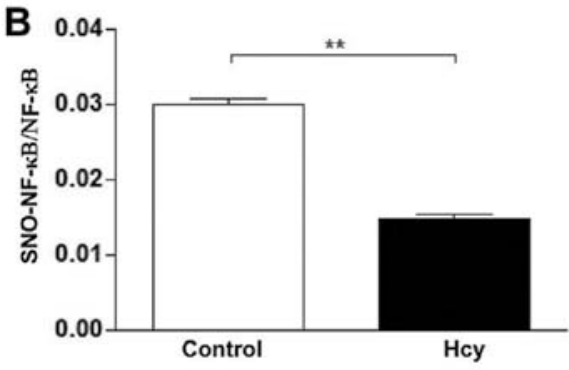

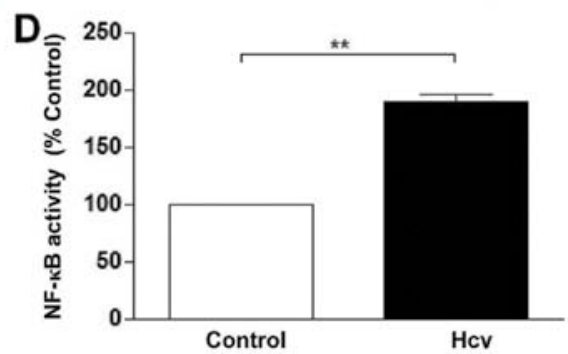

Figure 7. Effect of homocysteine (Hcy) on S-nitrosylation and the activity of nuclear factor $\kappa \mathrm{B}(\mathrm{NF}-\kappa \mathrm{B})$. Human umbilical vein endothelial cells (HUVECs) were treated with $2 \mathrm{mM}$ Hcy for $24 \mathrm{~h}$. The cell lysates were treated using BST. (A and B) S-nitrosylated NF- $\kappa$ B was purified by affinity chromatography using streptavidin-agarose and analyzed by western blot analysis using an anti-p65 antibody. (C and D) Activity of NF- $\kappa \mathrm{B}$ was detected by electrophoretic mobility shift assay (EMSA). The specificity of the bands was confirmed by supershifting with specific antibodies against p65 and p50 and by competition with specific cold competitor $\left(100 \mathrm{x}\right.$ SPC) and non-specific cold competitor $\left(100 \mathrm{x}\right.$ NSC) . The means \pm SEM of three independent experiments are presented. ${ }^{* *} \mathrm{P}<0.01$.

S-nitrosylation is a reversible covalent bonding to the $\mathrm{SH}$ group of a reactive cysteine on the target protein (2). Protein S-nitrosylation is also a ubiquitous post-translational modification that is responsible for a broad spectrum of biological functions. In endothelial cells, S-nitrosylation is able to affect cellular metabolic processes and regulate 

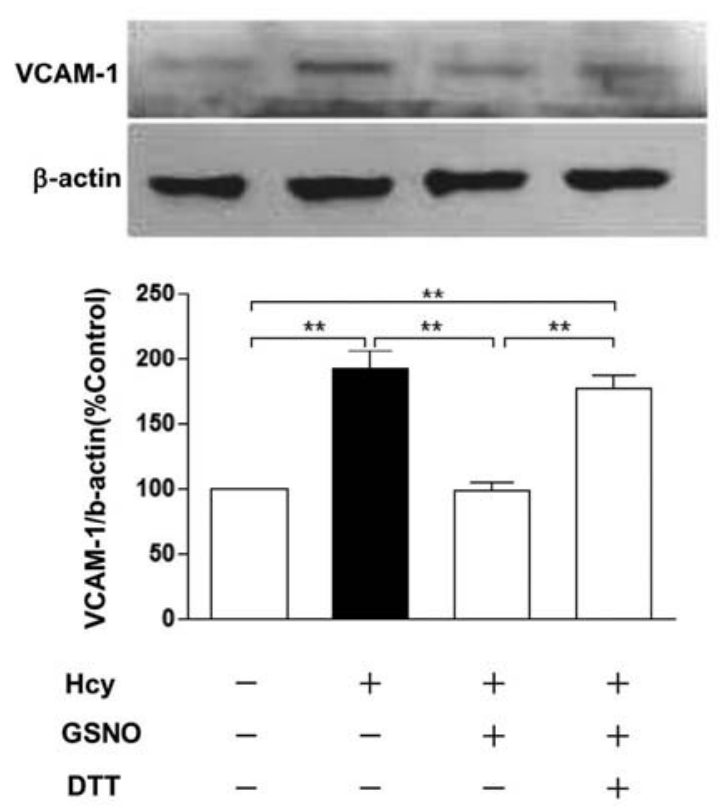

Figure 8. Effect of homocysteine (Hcy) on vascular cell adhesion molecule-1 (VCAM-1) in human umbilical vein endothelial cells (HUVECs). HUVECs were treated with $2 \mathrm{mM}$ Hcy and treated or not treated with GSNO $(100 \mu \mathrm{M})$ for $24 \mathrm{~h}$. A portion of the cells was incubated with DL-dithiothreitol (DTT) $(1 \mathrm{mM})$ for $10 \mathrm{~min}$ and washed twice. The cells were cultured in M199 medium containing $2 \%$ fetal bovine serum (FBS) for $6 \mathrm{~h}$, and after washing twice, VCAM-1 expression was detected by western blot analysis. The means \pm SEM of three independent experiments are presented. ${ }^{* *} \mathrm{P}<0.01$.

vascular functions such as inflammation $(6,9)$, apoptosis (8), permeability (20), migration (21), cell growth (22), and vascular stiffness (23). Over $100 \mathrm{~S}$-nitrosylation proteins have been identified in endothelial cells, many of which are important in regulating endothelial functions (24). For example, S-nitrosylation can inhibit the activity of nicotinamide adenine dinucleotide, whereas it promotes the activity of thioredoxin-1, thus resulting in the prevention of oxidative stress in endothelial cells (5). S-nitrosylation of p65 or p50 inhibits $\mathrm{NF}-\kappa \mathrm{B}$ activation, and suppresses inflammation in endothelial cells (9). Hcy decreases the levels of NO and induces endothelial dysfunction (13). In the present study, the levels of protein S-nitrosylation were reduced by Hcy in HUVECs, whereas the endogenous NO donor GSNO was able to rescue this reduction of protein S-nitrosylation. Thus, Hcy reduces the levels of protein S-nitrosylation by decreasing the levels of NO in endothelial cells. It was emphasized earlier that low Hcy does not evidently attenuate protein S-nitrosyaltion in a short period of time in vitro. However, when the concentration of Hcy was $>0.5 \mathrm{mM}$, the degree of attenuation regarding S-nitrosylation protein was only marginally deepened. HHcy also decreased endothelium S-nitrosyaltion in a rat model. Although plasma Hcy concentration is low, chronic plasma Hcy stimulation on the endothelium over a long period of time may be a major cause.

Oxidative stress has been widely accepted as an important mechanism of Hcy-induced endothelial dysfunction and damage (25). Hcy leads to the accumulation of ROS (26). We found that Hcy significantly increased the levels of ROS, however, this effect was blocked by VitC and NAC.
In addition, VitC and NAC reversed the reduction of protein S-nitrosylation induced by Hcy, suggesting that Hcy reduces protein S-nitrosylation levels due to the excessive generation of ROS. We also observed that Hcy decreases the production of NO, which is consistent with previous studies (26). Moreover, the reduction of NO is blocked by VitC. We hypothesized that Hcy reduces protein S-nitrosylation through the reduction of NO release which is induced by the overproduction of ROS. Under certain circumstances, ROS can also induce the dephosphorylation of Akt at Ser-473 (26), followed by Akt inactivation, leading to the dephosphorylation of eNOS at Ser-1177 and the reduction of NO generation in endothelial cells (27). Hcy did not affect the expression levels of Akt and eNOS, but decreased the levels of phosphorylated Akt at Ser-473 and eNOS at Ser-1177 in endothelial cells (19). We also found that Hcy may affect the phosphorylation of the Akt and eNOS proteins. Athough incubation with VitC increased the phosphorylation levels of Akt and eNOS proteins, the reduced levels of phosphorylation protein in Hcy-treated cells did not restore the control levels following incubation with VitC. This indicates that decreased phosphorylation levels of the Akt and eNOS proteins did not solely occur due to the accumulation of ROS. Furthermore, we found that the protein S-nitrosylation that was partially restored by VitC was abrogated by LY294002 and L-NAME in the endothelial cells treated with Hcy. Therefore, the protein S-nitrosylation inhibitory effect of Hcy may be associated with increased ROS levels together with a blockage of the Akt/ eNOS/NO pathway.

$\mathrm{NF}-\kappa \mathrm{B}$ is important in regulating endothelial pro-inflammatory genes, including VCAM-1 (28), while Hcy activates $N F-\kappa B$ via oxidative stress (29). Accumulating evidence suggests that NF- $\kappa \mathrm{B}$ activity is inhibited by $\mathrm{S}$-nitrosylation. The p65 component of NF- $\kappa \mathrm{B}$ is a target for S-nitrosylation, which inhibits its translocation into the cell nucleus (30). The p50

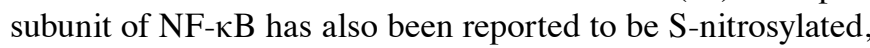
causing the inhibition of its binding to DNA $(7,9,31)$. In addition, inhibitory $\kappa \mathrm{B}$ kinase, the catalytic subunit required for the activation of $\mathrm{NF}-\kappa \mathrm{B}$, was $\mathrm{S}$-nitrosylated, resulting in loss of its activity (32). Thus, NO attenuated NF- $\kappa \mathrm{B}$ activity through a diverse $\mathrm{S}$-nitrosylation pathway. Hcy decreased the levels of cytoplasm S-nitrosylated p65, which may inhibit the translocation of $\mathrm{NF}-\kappa \mathrm{B}$ into the nucleus. GSNO rescued Hcy-induced decrease in levels of cytoplasm S-nitrosylated p65. Furthermore, Hcy enhanced the activity of $\mathrm{NF}-\kappa \mathrm{B}$, suggesting that inhibition of S-nitrosylated p65 is one of numerous pathways that activate $\mathrm{NF}-\kappa \mathrm{B}$ following treatment with Hcy. Consequently, the protein expression of VCAM-1 was also enhanced.

In conclusion, findings of the present study demonstrate that Hcy attenuates protein S-nitrosylation in endothelial cells and rat aortas. This may be associated with increased levels of ROS and a blockage of the Akt/eNOS/NO pathway and promotion of inflammation. This finding provides new insight into HHcy-induced vascular damage.

\section{Acknowledgements}

This study was partly supported by the National Natural Science Foundation of China (81070250) and by a Public Service Platform Grant of Shaanxi Province (2010FWPT-1). 


\section{References}

1. Martínez-Ruiz A and Lamas S: Signalling by NO-induced protein S-nitrosylation and S-glutathionylation: convergences and divergences. Cardiovasc Res 75: 220-228, 2007.

2. Lima B, Forrester MT, Hess DT and Stamler JS: S-nitrosylation in cardiovascular signaling. Circ Res 106: 633-646, 2010.

3. Chakrabarti S, Lekontseva O, Peters A and Davidge ST: 17beta-Estradiol induces protein S-nitrosylation in the endothelium. Cardiovasc Res 85: 796-805, 2010.

4. Sun J, Picht E, Ginsburg KS, Bers DM, Steenbergen C and Murphy E: Hypercontractile female hearts exhibit increased S-nitrosylation of the L-type Ca2t channel alpha1 subunit and reduced ischemia/reperfusion injury. Circ Res 98: 403-411, 2006.

5. Selemidis S, Dusting GJ, Peshavariya H, Kemp-Harper BK and Drummond GR: Nitric oxide suppresses NADPH oxidase-dependent superoxide production by S-nitrosylation in human endothelial cells. Cardiovasc Res 75: 349-358, 2007.

6. Kang-Decker N, Cao S, Chatterjee S, Yao J, Egan LJ, Semela D, Mukhopadhyay D and Shah V: Nitric oxide promotes endothelial cell survival signaling through S-nitrosylation and activation of dynamin-2. J Cell Sci 120: 492-501, 2007.

7. Marshall HE and Stamler JS: Inhibition of NF-kappa B by S-nitrosylation. Biochemistry 40: 1688-1693, 2001.

8. Hoffmann J, Haendeler J, Zeiher AM and Dimmeler S: TNFalpha and oxLDL reduce protein S-nitrosylation in endothelial cells J Biol Chem 276: 41383-41387, 2001.

9. Wadham C, Parker A, Wang L and Xia P: High glucose attenuates protein S-nitrosylation in endothelial cells: role of oxidative stress. Diabetes 58: 2715-2721, 2007.

10. Hoffmann J, Dimmeler S and Haendeler J: Shear stress increases the amount of S-nitrosylated molecules in endothelial cells: important role for signal transduction. FEBS Lett 551: 153-158, 2003.

11. Chen SC. Huang B, Liu YC, Shyu KG, Lin PY and Wang DL: Acute hypoxia enhances proteins' S-nitrosylation in endothelial cells. Biochem Biophys Res Commun 377: 1274-1278, 2008.

12. Hankey GJ and Eikelboom JW: Homocysteine and vascular disease. Lancet 354: 407-413, 1999.

13. Suematsu N, Ojaimi C, Kinugawa S, et al: Hyperhomocysteinemia alters cardiac substrate metabolism by impairing nitric oxide bioavailability through oxidative stress. Circulation 115: 255-262, 2007.

14. Ungvari Z, Sarkadi-Nagy E, Bagi Z, Szollár L and Koller A: Simultaneously increased TxA(2) activity in isolated arterioles and platelets of rats with hyperhomocysteinemia. Arterioscler Thromb Vasc Biol 20: 1203-1208, 2000.

15. Forrester MT, Foster MW, Benhar M and Stamler JS: Detection of protein S-nitrosylation with the biotin-switch technique. Free Radic Biol Med 46: 119-126, 2009.

16. Ota H, Eto M, Kano MR, Ogawa S, Iijima K, Akishita $M$ and Ouchi Y: Cilostazol inhibits oxidative stress-induced premature senescence via upregulation of Sirtl in human endothelial cells. Arterioscler Thromb Vasc Biol 28: 1634-1639, 2008.

17. Wu Y, Zhang W, Liu W, Zhuo X, Zhao Z and Yuan Z: The double-faced metabolic and inflammatory effects of standard drug therapy in patients after percutaneous treatment with drug-eluting stent. Atherosclerosis 215: 170-175, 2011.
18. Michaud SE, Dussault S, Groleau J, Haddad P and Rivard A: Cigarette smoke exposure impairs VEGF-induced endothelial cell migration: role of $\mathrm{NO}$ and reactive oxygen species. J Mol Cell Cardiol 41: 275-284, 2006

19. Lan TH, Xu ZW, Wang Z, Wu YL, Wu WK and Tan HM: Ginsenoside $\mathrm{Rb} 1$ prevents homocysteine-induced endothelial dysfunction via PI3K/Akt activation and PKC inhibition. Biochem Pharmacol 82: 148-155, 2011.

20. Thibeault S, Rautureau Y, Oubaha M, Faubert D, Wilkes BC, Delisle $\mathrm{C}$ and Gratton JP: S-nitrosylation of beta-catenin by eNOS-derived NO promotes VEGF-induced endothelial cell permeability. Mol Cell 39: 468-476, 2010.

21. Pi X, Wu Y, Ferguson JE III, Portbury AL and Patterson C: SDF-1alpha stimulates JNK3 activity via eNOS-dependent nitrosylation of MKP7 to enhance endothelial migration. Proc Natl Acad Sci USA 106: 5675-5680, 2009.

22. Oliveira CJ, Curcio MF, Moraes MS, Tsujita M, Travassos LR, Stern A and Monteiro HP: The low molecular weight S-nitrosothiol, S-nitroso-N-acetylpenicillamine, promotes cell cycle progression in rabbit aortic endothelial cells. Nitric Oxide 18: 241-255, 2008.

23. Santhanam L, Tuday EC,Webb AK, et al: Decreased S-nitrosylation of tissue transglutaminase contributes to age-related increases invascular stiffness. Circ Res 107: 117-125, 2010

24. Martínez-Ruiz A and Lamas S: Detection and proteomic identification of S-nitrosylated proteins in endothelial cells. Arch Biochem Biophys 423: 192-199, 2004.

25. McCully KS: Chemical pathology of homocysteine. IV. Excitotoxicity, oxidative stress, endothelial dysfunction, and inflammation. Ann Clin Lab Sci 39: 219-232, 2009.

26. Chavakis E, Dernbach E, Hermann C, Mondorf UF, Zeiher AM and Dimmeler S: Oxidized LDL inhibits vascular endothelial growth factor-induced endothelial cell migration by an inhibitory effect on the Akt/endothelial nitric oxide synthase pathway. Circulation 103: 2102-2107, 2001.

27. Dimmeler S, Fleming I, Fisslthaler B, Hermann C, Busse R and Zeiher AM: Activation of nitric oxide synthase in endothelial cells by Akt-dependent phosphorylation. Nature 399: 601-605, 1999.

28. Carluccio MA, Ancora MA, Massaro M, et al: Homocysteine induces VCAM-1 gene expression through NF-kappaB and NAD(P)H oxidase activation: protective role of Mediterranean diet polyphenolic antioxidants. Am J Physiol Heart Circ Physiol 293: H2344-H2354, 2007.

29. Au-Yeung KK, Woo CW, Sung FL, Yip JC, Siow YL and O K: Hyperhomocysteinemia activates nuclear factor-kappaB in endothelial cells via oxidative stress. Circ Res 94: 28-36, 2004.

30. Kelleher ZT, Matsumoto A, Stamler JS and Marshall HE: NOS2 regulation of NF-kappaB by S-nitrosylation of p65. J Biol Chem 282: 30667-30672, 2007.

31. Marshall HE, Hess DT and Stamler JS: S-nitrosylation: physiological regulation of NF-kappaB. Proc Natl Acad Sci USA 101: 8841-8842, 2004.

32. Reynaert NL, Ckless K, Korn SH, et al: Nitric oxide represses inhibitory kappaB kinase through S-nitrosylation. Proc Natl Acad Sci USA 101: 8945-8950, 2004. 